\title{
FUSION AND BREAKUP IN REACTIONS INVOLVING WEAKLY BOUND NUCLEI
}

A. Szanto de Toledo ${ }^{1}$, F.A. Souza ${ }^{1}$, C. Beck ${ }^{2}$, S.J. Sanders ${ }^{3}$, M.G. Munhoz ${ }^{1}$, J. Takahashi $^{1}$, N. Carlin ${ }^{1}$, A.A.P. Suaide ${ }^{1}$, M.M. de Moura ${ }^{1}$ and E.M. Szanto ${ }^{1}$

${ }^{1}$ Instituto de Física - Universidade de São Paulo, Deptartamento de Física Nuclear, Laboratório Pelletron, CP 66318, 05315-970, São Paulo - SP, Brazil

${ }^{2}$ Centre National de la Recherche Scientifique Université Louis Pasteur, 23 rue du Loess, B.P. 28, F-67037 Strasbourg Cedex 2, France and ${ }^{3}$ University of Kansas, Lawrence, KS 66045, USA

(Dated: September 16, 2003) 
The effect of collective degrees of freedom on the fusion process has been extensively investigated over the past few years [1]. A significant enhancement of the sub-barrier fusion cross section is often found as compared to the predictions of one-dimensional barrier penetration models. This enhancement is understood in terms of dynamical processes involving couplings to collective inelastic excitations of the target and/or projectile. A precise determination of the "barrier distributions" leading to the enhancement requires an understanding of the dominant channels that couple to the fusion channel[2]. However, in the case of reactions where at least one of the colliding nuclei has a sufficiently low binding energy so that breakup becomes an important process, conflicting experimental and theoretical results have been reported [3-11]. The many questions regarding the influence of breakup become more relevant with the recent availability of radioactive beams and the renewed interest in super-heavy element formation. Radioactive ion beams are likely to produce intense breakup yields. In the fusion processes, and more specifically in the fusion of weakly bound nuclei, as likely to be used in super-heavy element studies, two different and independent processes can be distinguished both experimentally and theoretically. One, denoted as "Complete Fusion" (CF), is associated with the capture of all of the projectile constituents by the target. The other, denoted as "Incomplete Fusion" (ICF) or Partial Fusion, occurs when part of the projectile is captured by the target and the remaining part escapes. "Total Fusion" is understood as the sum of these two processes $(\mathrm{CF}+\mathrm{ICF})$. In order to avoid misinterpretations, a clear definition of the experimental and theoretical quantities being compared is essential. This is the main difficulty in comparing data and/or calculations from different authors. Depending on the theoretical approach, different results are achieved. If fusion occurs incoherently, i.e., with possible breakup of the incoming particle $[4,5]$, the survival probability of this particle prior to fusion, is lower than unity, resulting in a decrease in the effective entrance-channel flux and a corresponding reduction of the fusion cross section. On the other hand, if the breakup channel is coupled coherently to the fusion channel, as expected in references $[3,6]$, entrance barrier fluctuations will lead to an effective lowering of this barrier, resulting in an overall cross section enhancement. Recent coupled-channel calculations based on the coupling to states in the continuum, which is taken into account by splitting the continuum into discrete bins, (CDCC) [7], predict the coexistence of both dynamical descriptions: a coherent process at sub-barrier energies leads to a cross section enhancement and, at higher energies, a significant loss of entrance-channel flux leads to an 
overall cross section suppression. A similar situation is found experimentally. In the case of heavy systems, two different situations are observed. Data for fusion reactions presented in reference [8-10] indicate the occurrence of a significant cross-section enhancement. On the other hand, in references [11] a suppression is observed. References [12] and [13] report the simultaneous occurrence of both effects in different energy regimes. In the case of light systems, a correlation between the "Fusion Inhibition Factor", FIF $=\sigma_{\text {fusion }} / \sigma_{\text {reaction }}$ and the binding energy has been observed [14-16]; ( $\sigma_{\text {fusion }}$ represents the experimental fusion cross section at the energy of two times the fusion barrier, and $\sigma_{\text {reaction }}$ represents the reaction cross section extracted from Optical Model fits to the elastic scattering- for details see ref. [14] and [15]) (see Fig. 1). However it has been found [15] that two factors contribute to FIF. One is the relative importance of the surface to core density distribution that contributes to fusion (which increases for very light nuclei) and another is related to the survival probability (which increases with binding energy). Fusion reactions involving medium weight nuclei were also investigated by means of the ${ }^{6,7} \mathrm{Li}+{ }^{59} \mathrm{Co}$ reactions [17]. Fusion cross sections are presented in Fig. 2 for the ${ }^{6} \mathrm{Li}$ and ${ }^{7} \mathrm{Li}$ induced reactions as well as the ratio $R=\sigma_{6}{ }_{L i} / \sigma^{\sigma_{L i}}$. (The ${ }^{7} \mathrm{Li}$ induced reaction is used as reference due to its higher binding energy $(2.47 \mathrm{MeV}$ ) when compared to $1.57 \mathrm{MeV}$ for ${ }^{6} \mathrm{Li}$ ). Results from coupled channel calculations for these systems are presented in Fig. 2. Predictions based on CDCC [18], shown in Fig. 3, indicate that no significant suppression is expected at energies above the barrier energies but, at sub-barrier energies, a fusion cross section enhancement is predicted due to the coupling of the breakup channel. A complete accounting for the coupling to the breakup channel, as well as the correct description of the reaction dynamics, requires the explicit measurement of yields leading to breakup itself. Some experiments have already addressed this issue [19-22]. In the present case of the ${ }^{6,7} \mathrm{Li}+{ }^{59} \mathrm{Co}$ reactions, we have initiated this investigation (see Fig. 4) and have identified the Li states involved in the breakup process.

Acknowledgments: This work was supported in part by the Conselho Nacional de DesenvolvimentoCientífico e Tecnológico $(\mathrm{CNPq})$ and Fundação de Amparo à Pesquisa do Estado de São Paulo (FAPESP)-Brasil.

[1] M. Dasgupta et al., Ann. Rev. Nucl. Part. Sci. 48(1998) 401 and references therein.

[2] N. Rowley et al., Phys. Lett. B254 (1991) 25. 
[3] C.H. Dasso et al Phys. Rev. C50, R12 (1994) and Nucl. Phys. A597,473(1996).

[4] L.F. Canto et al Phys. Rev. C 52, 1 (1995).

[5] N. Takigawa et al, Phys. Rev. C47, R2470 (19930).

[6] K. Hagino et al, Phys. Rev. C61, 037602 (2000).

[7] A. Diaz-Torres and I.J. Thompson, Phys. Rev. C65, 024606(2002).

[8] K. Yoshida et al., PLB389, 457 (1996).

[9] J.J. Kolata et al., Phys. Rev. Lett 81, 4580 (1988).

[10] M. Trotta et al., Phys. Rev. Lett84, 2342 (2000).

[11] K.E. Rehm et al, Phys. Rev. Lett. 81, 3341 (1998).

[12] N.Dasgupta et al, Phys. Rev. Lett 82,1395 (1999) and Phys. Rev. C66, 041602R (2002).

[13] V.Tripathi et al, Phys. Rev. Lett 88 (2001) 172701-1.

[14] J.Takahashi et al, Phys.Rev.Lett.78, 30 (1997).

[15] A.Szanto de Toledo et al, Nucl.Phys. A679, 175 (2000) and R.Cabezas et al, Phys. Rev. C60, $067602(1999)$.

[16] R.M. Anjos et al. Phys. Lett. 534B, 295 (2002).

[17] C. Beck et al., PRC 67 (2003) 54602.

[18] A. Diaz-Torres et al, Phys. Rev. C ( to be published and priv. com.).

[19] D.J. Hinde et al. , PRL 89 (2002) 272701.

[20] K. Rusek et al., PRC 67 (2003) 41604.

[21] J.F. Liang et al., PRC 67 (2003) 44603.

[22] C.Signorini et al., PRC 67 (2003) 44607. 


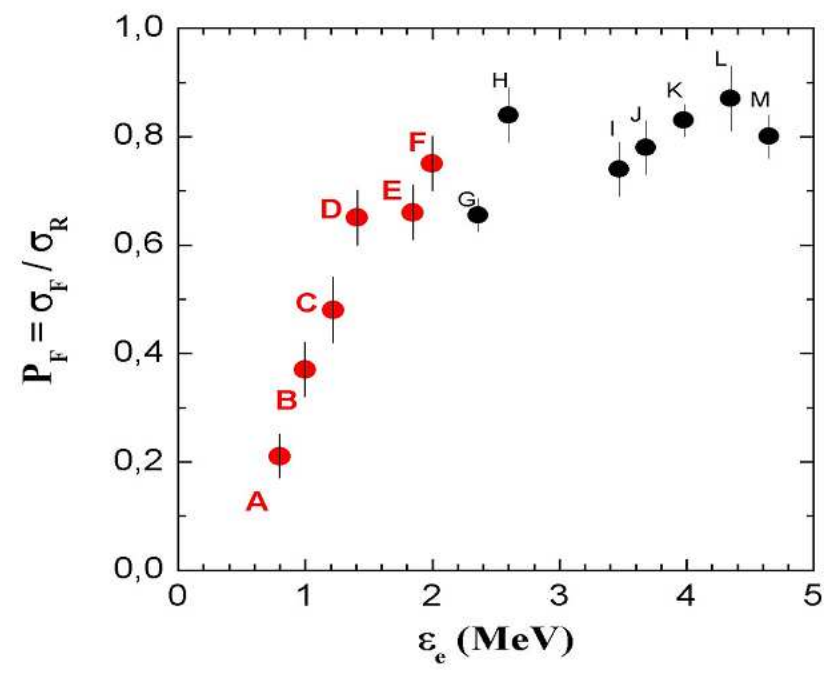

FIG. 1: Fusion Inhibition Factor $F I F=\sigma_{\text {fusion }} / \sigma_{\text {reaction }}$ (for a set of reactions identified in reference [14]) as a function of the binding energy of the colliding nuclei.

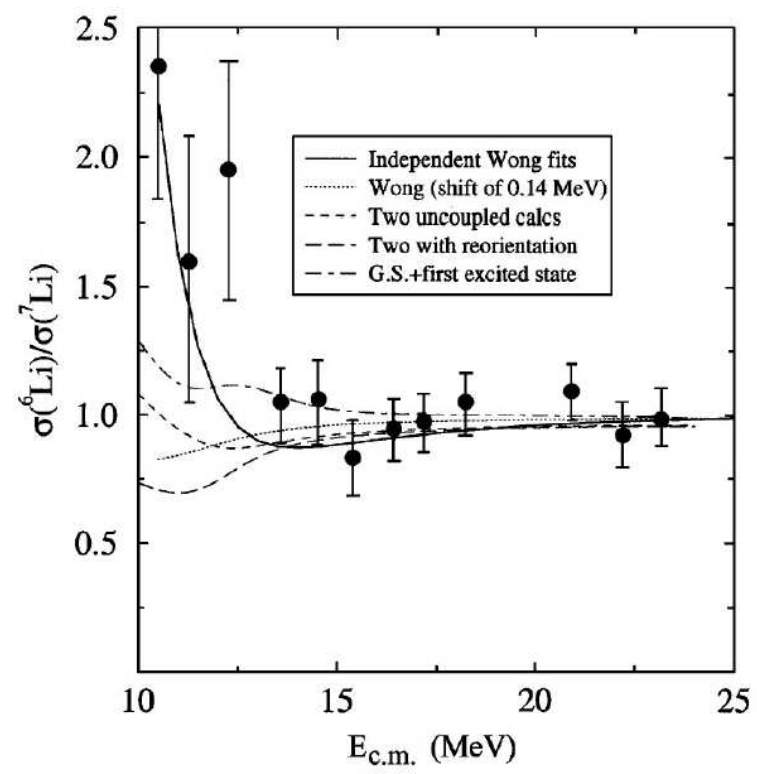

FIG. 2: Energy dependence of the ratio $R=\sigma_{6}{ }_{L i} / \sigma_{7}{ }_{L i}$ between the fusion cross sections for ${ }^{6} \mathrm{Li}$ and ${ }^{7} \mathrm{Li}$ induced reactions. Error bars reflect the large systematic errors. The solid and dashed curves correspond to the case of fits of the experimental data to Single Barrier Penetration Model. Dotted curves correspond to two uncoupled CCFULL calculations [17] with and without reorientation effects. 


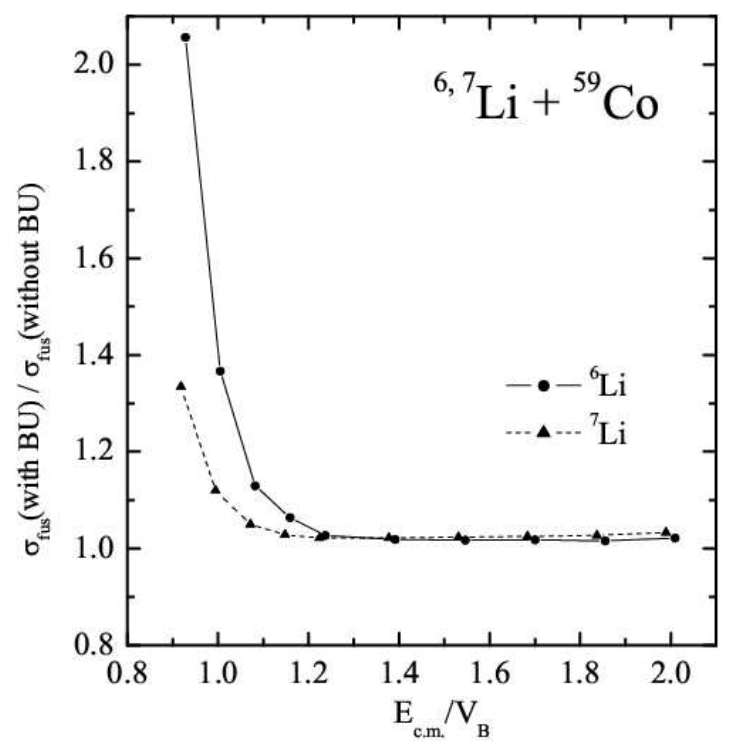

FIG. 3: Total fusion excitation functions for ${ }^{6} \mathrm{Li}+{ }^{59} \mathrm{Co}$ (full dots) and ${ }^{7} \mathrm{Li}+{ }^{59} \mathrm{Co}$ (full triangles), which are normalized with the cross sections in absence of couplings to breakup channels. For each reaction, the incident energy is normalized with the Coulomb barrier VB of the bare potential [18]. The curves are drawn to guide the eyes.

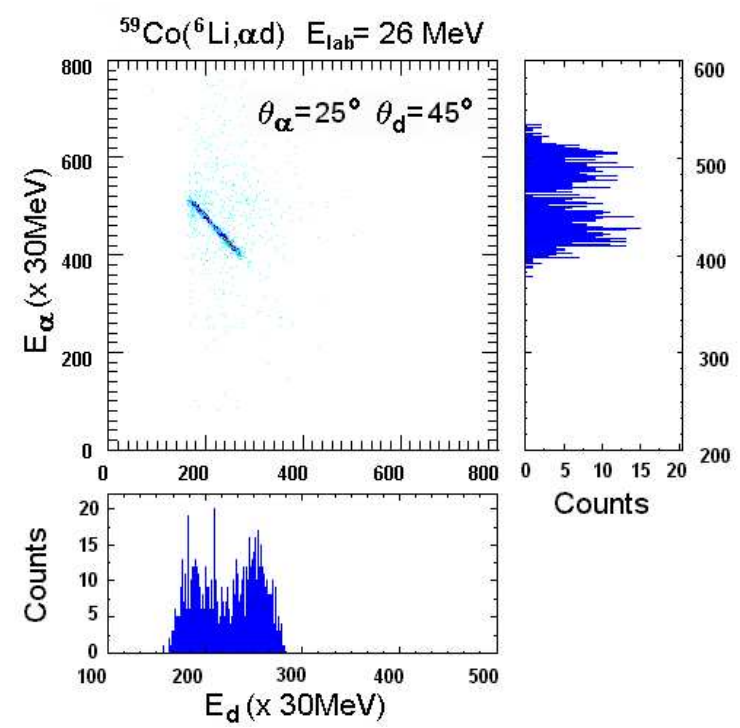

FIG. 4: Energy correlation plots $\mathrm{E}_{-} \alpha$ vs $\mathrm{E}_{d}$, for $\alpha$ particles and deuterons, detected in coincidence for the ${ }^{59} \mathrm{Co}\left({ }^{6} \mathrm{Li}, \alpha \mathrm{d}\right)$ reaction. The strait line in this plane $\left(\mathrm{E}_{\alpha}+\mathrm{E}_{d}=\right.$ Constant $)$ is associated to a well defined Q-value process. Projections of the $\mathrm{E}_{\alpha}$ (right panel) and $\mathrm{E}_{d}$ (lower panel) are also shown. 